\title{
Retinal pigment epithelium: lysosomal enzymes and aging
}

The neural retina and its closely apposed layer of pigment epithelial cells form a functional unit essential for vision. In cases of retinal detachment or other conditions where this apposition is disturbed, vision is severely compromised. Owing to their strategic location and their specialised transport properties, the retinal pigment epithelial (RPE) cells provide the principal mechanism for transfer of nutrients between the choriocapillaris and the photoreceptors. They are also the principal storage depot for vitamin $\mathrm{A}$ in the eye and these cells supply the essential visual cycle intermediate, 11-cis retinal, to the photoreceptors for regeneration of rhodopsin. In some species the RPE cells are responsible for the synthesis and secretion of chondroitin sulphate proteoglycans of the interphotoreceptor matrix.

In addition to the foregoing functions, RPE cells play a major and essential role in the outer segment renewal process. ${ }^{1-3}$ Rhodopsin, phospholipids, and other components of the disc membranes are synthesised in the endoplasmic reticulum of the inner segments and are afterwards transported to the base of the outer segments for assembly into new discs. These components are then displaced distally along the length of the rod outer segment, and finally, small packets of terminal discs are shed. In this way, despite the continuous formation of new discs, the photoreceptors maintain constant axial lengths. The outer segment tips shed in this process are rapidly phagocytosed by the pigment epithelium. This is not a random process, rather the greatest burst of shedding and phagocytic activity occurs after the onset of morning light. ${ }^{4}$ The whole process is light entrained and triggered by circadian rhythm. ${ }^{5}$ In most species, outer segment membranes are renewed every 10-14 days, and each RPE cell ingests 2000-4000 discs per day. ${ }^{6}$ Expressed differently, each RPE cell must not only phagocytose, but also degrade nearly $10 \%$ of adjacent photoreceptors each day.

Degradation of these ingested outer segments is achieved through a highly developed phagolysosomal system first described in human RPE by Feeney. ${ }^{7}$ Shortly after entering the RPE cell, the ingested outer segment discs rapidly fuse with primary lysosomes to form phagosomes, ${ }^{67}$ which are then converted to phagolysosomes in a two step process involving interactions between two different populations of lysosomes. ${ }^{8}$ In the first, small lysosomes fuse directly with phagosomes to form the characteristic phagolysosomes; independently of this, small lysosomes also fuse with one another forming larger organelles which, at a somewhat later stage, also interact with phagosomes. Interchange of materials, or injection of lysosomal enzymes, is thought to occur during these two types of fusion. The two processes are independent of one another and are driven by mechanisms that are both circadian and light triggered.

The lamellar discs in the phagolysosomes are thought to be partially degraded by RPE lysosomal enzymes such as $\alpha$-mannosidase, ${ }^{9}$ cathepsin $D,{ }^{10}$ and phospholipase $A,{ }^{11}$ either singly or in concert. Although a whole battery of other acid hydrolases are known to be present in the RPE lysosomes, their activities are only measurable by use of artificial substrates. Activities toward physiological substrates - for example, outer segment discs, can sometimes be demonstrated under carefully defined experimental conditions: Certain pitfalls in assessing lysosomal hydrolase activities should be pointed out. The measurements are made as total activity using synthetic substrates; to obtain specific activities, total activity is corrected for either protein content (used by most investigators) or number of cells in the tissue sample (used by Boulton et al in this issue). This can lead to a wide range of values for specific activities if there are large variations in either protein content or cell count in the individual samples.

In most cells throughout the body, lysosomal acid hydrolases are responsible for the degradation of a variety of macromolecules such as proteins, lipids, complex carbohydrates, and nucleic acids. These substances are present in organelles derived from both exogenous and endogenous sources, the latter known as autophagic vacuoles. ${ }^{12}$ Similar macromolecules, as well as outer segment membranes, are undoubtedly acted upon by RPE lysosomal enzymes, but the sequence of these putative degradative steps is not clear. It has, however, been established that the major end products of lysosomal enzyme action in the phagolysosomal system of RPE cells are lipofuscin granules, autofluorescent particles that accumulate in an age-related manner in the cytoplasm of RPE cells. ${ }^{1314}$ Other components of the phagolysosomal system are melanolipofuscin and melanolysosomes. Residual bodies, thought to represent incomplete breakdown products of phagolysosomes, ${ }^{73}$ and drusen, extracellular excrescences of RPE cells that are common in the elderly population, are thought to arise as additional end products of the phagolysosomal system.

The RPE cell population is not homogeneous, and it is now recognised that lysosomal acid hydrolases show regional differences in their activity. Boulton et al, in this issue of the journal, report higher activities of acid phosphatase and cathepsin D in macular than in peripheral regions of human RPE. These findings are consistent with the higher RPE cell density in foveal regions than in equatorial regions of human RPE. ${ }^{15}$ There are also some similarities in animal species; cathepsin D activity is higher in the 'macular' posterior centralis areas of canine ${ }^{16}$ and bovine ${ }^{17}$ RPE than in cells originating from peripheral regions. Nevertheless, the activities of other canine RPE enzymes such as acid phosphatase, $B$-glucuronidase, and $N$-acetyl- $B$-glucosaminidase have been reported to be higher in cells derived from the peripheral region than in those from central areas. ${ }^{16}$ Photoreceptor density per RPE cell does not vary significantly in various regions of the canine eye, hence the variations in RPE enzyme activities appear to be unrelated to differences in photoreceptor cell distribution.

It is common knowledge that with aging the human retina undergoes functional, structural, and physiological changes whose basis is not well understood. Many important aspects of senescence in the neural retina and in RPE cells have been discussed in two excellent review articles. ${ }^{18} 19$ The RPE cells are for the most part non-mitotic cells, and the continuous phagocytic load imposed upon them throughout life leads to a striking age-related accumulation, from about age 40 , of autofluorescent lipofuscin granules. ${ }^{718}$ With advancing age, these particles may in some cases fill almost the entire RPE cell. ${ }^{14}$ This accumulation is especially striking in the macular region. Other age-related structural changes in Bruch's membrane as well as drusen formation have been studied extensively; the latter are not sight threatening, but changes in Bruch's membrane, possibly initiated by (abnormal) substances secreted by aging RPE cells, lead to changes in structure and elasticity which ultimately interfere with the transport of essential nutrients to the photoreceptors. ${ }^{18}$

A detailed study of 35 human eyes spanning a period from the second to the ninth decade of life showed no selective agerelated loss of either photoreceptors or RPE; instead, it was found that equatorial cones and RPE cells decrease at uniform rates throughout this time. ${ }^{15}$ The same study showed that there were no significant differences in cone or RPE densities in the foveal region as a function of age. It was 
concluded that there is a parallel loss of both photoreceptors and RPE cells during aging, the loss of photoreceptors being more pronounced in the equatorial region than at the fovea.

Finally, the question of age-related changes in RPE lysosomal enzyme activities should be addressed. Although it is tempting to speculate that malfunctioning enzymes, especially in the macular region, could be involved in the pathogenesis of disorders such as age-related macular degeneration, there are only a few isolated reports examining this possibility. One study on enzyme measurements in cultured RPE cells from donors ranging in age from 19 to 80 years suggests a selective age-related decrease in $\alpha$-mannosidase activity compared with other enzymes which were within the normal range. ${ }^{9}$ In contrast, in their paper Boulton et al report that the activities of two lysosomal enzymes, acid phosphatase and cathepsin $D$, are increased in an age-related manner in all regions of the RPE. Further investigations are needed to resolve these conflicting findings.

Hadassah University Hospital,

ELAINE R BERMAN

PO Box 23099,

Jerusalem, Israel

1 Young RW, Bok D. Participation of the retinal pigment epithelium in the rod outer segment renewal process. F Cell Biol 1969; 42: 392-403.

2 Bok D. Retinal photoreceptor-pigment epithelium interactions. Invest Ophthalmol Vis Sci 1985; 26: 1659-94.

3 Berman ER. Biochemistry of the eye. New York: Plenum, 1991: 380-406.
4 LaVail MM. Rod outer segment disk shedding in rat retina: relationship to cyclic lighting. Science 1976; 194: 1071-4.

5 Hollyfield JG, Basinger SF. Photoreceptor shedding can be initiated within the eye. Nature 1978; 274: 794-6.

6 Young RW. Visual cells and the concept of renewal. Invest Ophthalmol Vis Sci 1976; 15: 700-25.

7 Feeney L. The phagolysosomal system of the pigment epithelium. A key to retinal disease. Invest Ophthalmol Vis Sci 1973; 12: 635-8.

8 Bosch E, Horwitz J, Bok D. Phagocytosis of outer segments by retinal pigment epithelium: phagosome-lysosome interaction. $\mathcal{F}$ Histochem Cytochem 1993; 41: 253-63.

9 Wyszynski RE, Bruner WE, Cano DB, Morgan KM, Davis CB, Sternberg P. A donor-age-dependent change in the activity of alpha-mannosidase in human cultured RPE cells. Invest Ophthalmol Vis Sci 1989; 30: 2341-7.

10 Regan CM, deGrip WJ, Daemen FJM, Bonting SL. Degradation of rhodopsin by a lysosomal fraction of retinal pigment epithelium. Biochemical aspects of the visual process. Exp Eye Res 1980; 30: 183-91.

11 Zimmerman WF, Godchaux W III, Belkin M. The relative proportions of lysosomal enzyme activities in bovine retinal pigment epithelium. Exp Eye Res 1983; 36: 151-8.

12 Berman ER. Lysosomal storage and transport disorders. In: Garner A, Klintworth GK, eds. Pathobiology of ocular disease. 2nd ed. New York: Marcel Dekker, 1994: chapter 26.

13 Feeney L. Lipofuscin and melanin of human retinal pigment epithelium. Fluorescence, enzyme cytochemical, and ultrastructural studies. Invest Ophthalmol Vis Sci 1978; 17: 583-600.

14 Wing GL, Blanchard GC, Weiter JJ. The topography and age relationship of lipofuscin concentration in the retinal pigment epithelium. Invest Ophthalmol is Sci 1978; 17: 601-7.

15 Gao H, Hollyfield JG. Aging of the human retina. Differential loss of neurons and retinal pigment epithelial cells. Invest Ophthalmol Vis Sci 1992; 33: 1-17.

16 Cabral L, Unger W, Boulton M, Lightfoot R, McKechnie N, Grierson I, et al. Regional distribution of lysosomal enzymes in the canine retinal pigment epithelium. Invest Ophthalmol Vis Sci 1990; 31: 670-6.

17 Burke JM, Twining SS. Regional comparisons of cathepsin D activity in bovine retinal pigment epithelium. Invest Ophthalmol Vis Sci 1988; 29: 1789-93.

18 Marshall J. The ageing retina: physiology or pathology. Eye 1987; 1: 282-95.

19 Boulton ME. Ageing of the retinal pigment epithelium. In: Osborne NN, Chader GJ, eds. Progress in retinal research. Vol 11. Oxford, New York, Seoul, Tokyo: Pergamon, 1991: 125-51. 